PROCEEDINGS OF THE

AMERICAN MATHEMATICAL SOCIETY

Volume 135, Number 12, December 2007, Pages 3887-3894

S 0002-9939(07)08923-X

Article electronically published on August 30, 2007

\title{
CHEBOTAREV-TYPE THEOREMS IN HOMOLOGY CLASSES
}

\author{
MARK POLLICOTT AND RICHARD SHARP
}

(Communicated by Jane M. Hawkins)

\begin{abstract}
We describe how closed geodesics lying in a prescribed homology class on a negatively curved manifold split when lifted to a finite cover. This generalizes a result of Zelditch in the case of compact hyperbolic surfaces.
\end{abstract}

\section{INTRODUCTION}

Given a compact manifold of negative curvature, there are geometric analogues of the Chebotarev Theorem in algebraic number theory due to Sunada 13] (cf. also Parry and Pollicott 8 for the generalization to Axiom A flows). More precisely, given a finite Galois cover of the manifold, these theorems describe the proportion of closed geodesics which lift in a prescribed way to the cover.

In this geometric setting, it is also natural to consider infinite covers, and, in particular, the number of closed geodesics lying in a prescribed homology class has been studied by Katsuda and Sunada [4, Phillips and Sarnak 9, Katsuda [3, Lalley [7] and Pollicott [10] (with generalizations to Anosov flows by Katsuda and Sunada [5] and Sharp [12]). In this note we shall combine these points of view, generalizing a result of Zelditch for hyperbolic Riemann surfaces [14.

Let $M$ be a compact smooth Riemannian manifold with negative curvature. Let $\widetilde{M}$ be a finite Galois covering of $M$ with covering group $G$. For a closed geodesic $\gamma$ on $M$, let $l(\gamma)$ denote its length, $\langle\gamma\rangle$ its Frobenius class in $G$ and $[\gamma]$ its homology class in $H=H_{1}(M, \mathbb{Z})$.

We shall examine how the closed geodesics lying in a fixed homology class $\alpha \in H$, split when lifted to $\widetilde{M}$. More precisely, for a conjugacy class $C$ in $G$, we study the asymptotics of

$$
\pi(T, \alpha, C)=\operatorname{Card}\{\gamma: l(\gamma) \leq T,[\gamma]=\alpha,\langle\gamma\rangle=C\} .
$$

The problem is complicated by the fact that, in general, $[\gamma]$ and $\langle\gamma\rangle$ are not independent quantities. This occurs if the abelian quotient group $G /[G, G]$ is nontrivial, since this group is also a quotient of $H$, the maximal abelian covering group of $M$. Let $\pi_{G}: G \rightarrow G /[G, G]$ and $\pi_{H}: H \rightarrow G /[G, G]$ be the natural projections. In particular, the image $\pi_{G}(C)$ of a conjugacy class $C \subset G$ is a single element in $G /[G, G]$ and if $\pi_{G}(C) \neq \pi_{H}(\alpha)$, then $\pi(T, \alpha, C)=0$, for all values of $T$.

On the other hand, we have the following result, which extends work of Zelditch for Riemann surfaces [14].

Received by the editors August 16, 2006 and, in revised form, September 1, 2006.

2000 Mathematics Subject Classification. Primary 37C27, 37C30, 37D40. 
Theorem 1. If $\pi_{G}(C) \neq \pi_{H}(\alpha)$, then $\pi(T, \alpha, C)$ is identically zero. If $\pi_{G}(C)=$ $\pi_{H}(\alpha)$, then

$$
\frac{\pi(T, \alpha, C)}{\pi(T, \alpha)} \rightarrow\left|\frac{G}{[G, G]}\right| \frac{|C|}{|G|} \text { as } T \rightarrow+\infty,
$$

where $\pi(T, \alpha)=\operatorname{Card}\{\gamma: l(\gamma) \leq T,[\gamma]=\alpha\}$.

Example. Let $G$ be a finite nonabelian nilpotent group. Then $[G, G] \neq G$. For definiteness, we can let $G=\{ \pm 1, \pm i, \pm j, \pm k\}$ be the quaternion group of eight elements. Then $[G, G]= \pm 1$. Let $\Gamma=\left\langle a_{1}, a_{2}, b_{1}, b_{2}: a_{1} b_{1} a_{1}^{-1} b_{1}^{-1} a_{2} b_{2} a_{2}^{-1} b_{2}^{-1}=1\right\rangle$ be a cocompact Fuchsian group. Define a homomorphism $\phi: \Gamma \rightarrow G$ by setting $\phi\left(a_{1}\right)=i, \phi\left(a_{2}\right)=j$ and $\phi\left(b_{1}\right)=\phi\left(b_{2}\right)=1$ and extending this to $\Gamma$. We can then define a normal subgroup by $\Gamma_{0}=\operatorname{ker}(\phi)$. If we set $M=\mathbb{H}^{2} / \Gamma_{0}$ and $\widetilde{M}=\mathbb{H}^{2} / \Gamma_{0}$, then $\widetilde{M}$ is a finite cover of $M$ with covering group $G$.

Let us consider a closely related problem. Consider the frame flow $f_{t}: \mathcal{F} M \rightarrow$ $\mathcal{F} M$ on the space of orthonormal frames above $M$. This is an $S O(n-1)$-extension for the geodesic flow. Changing notation slightly, let $\gamma$ be a periodic orbit of the geodesic flow, to which we associate a holonomy $\Theta(\gamma) \in S O(n-1)$ which comes from a reference frame being transported around $\gamma$. This is defined up to conjugacy. In 8 it was shown that the holonomies were equidistributed on $S O(n-1)$. The following shows that the corresponding result holds for geodesics in a fixed homology class. (Recall that a class function is a function that is constant on conjugacy classes.)

Theorem 2. Let $F: S O(n-1) \rightarrow \mathbb{R}$ be a class function. Then

$$
\frac{1}{\pi(T, \alpha)} \sum_{\substack{l(\gamma) \leq T \\ \gamma=\alpha}} F(\Theta(\gamma)) \rightarrow \int F d \lambda, \quad \text { as } T \rightarrow+\infty,
$$

where $\lambda$ denotes the Haar measure on $S O(n-1)$.

\section{Preliminaries}

Let $M$ be a compact smooth manifold equipped with a Riemannian metric of negative curvature and let $X$ denote its universal cover. (In the special case where $M$ is a surface with constant curvature $-1, X$ is the hyperbolic plane $\mathbb{H}$.) Then there is a discrete group of isometries $\Gamma \cong \pi_{1}(M)$ of $X$ such that $M=X / \Gamma$. Now let $\Gamma_{0}$ be a normal subgroup of $\Gamma$ with finite index. Then $\widetilde{M}=X / \Gamma_{0}$ is a finite (Galois) covering of $M$, with covering group $G=\Gamma / \Gamma_{0}$ (i.e., $G$ acts transitively on the fibres above each point in $M$ ).

There is a natural dynamical system, the geodesic flow, associated to $M$. Let $S M$ denote the unit-tangent bundle of $M$ and, for $(x, v) \in S M$, let $\gamma: \mathbb{R} \rightarrow M$ be the unique unit-speed geodesic with $\gamma(0)=x$ and $\dot{\gamma}(0)=v$. Then the geodesic flow $\phi_{t}: S M \rightarrow S M$ is defined by $\phi_{t}(x, v)=(\gamma(t), \dot{\gamma}(t))$, and we shall write $h$ for its topological entropy. There is a one-to-one correspondence between periodic $\phi$-orbits and directed closed geodesics on $M$. The fact that $M$ is negatively curved ensures that the geodesic flow is an Anosov flow and that $h>0$. This will enable us to use results proved in the context of Anosov flows in this setting.

We shall make use of $L$-functions defined with respect to certain representations of $\Gamma=\pi_{1}(M)$. Let $\rho: \Gamma \rightarrow U(d)$ be a unitary representation of $\Gamma$. We define an 
$L$-function $L(s, \rho)$ by the product formula

$$
L(s, \rho)=\prod_{\gamma} \operatorname{det}\left(I-\rho(\langle\langle\gamma\rangle\rangle) e^{-s l(\gamma)}\right)^{-1},
$$

where the product is taken over all (prime) closed geodesics $\gamma$ on $M$ and $\langle\langle\gamma\rangle\rangle$ denotes the homotopy class of $\gamma$. Provided $\operatorname{Re}(s)$ is sufficiently large, this will converge to an analytic function. We shall prove Theorem 1 by studying the behaviour of $L(s, \rho)$ for a restricted class of representations.

We shall be interested in how closed geodesics on $M$ lift to $\widetilde{M}$. There are a countable infinity of closed geodesics on $M$; we shall denote a typical one by $\gamma$ and its length by $l(\gamma)$. Each such $\gamma$ has $n=|G|$ lifts $\gamma_{1}, \ldots, \gamma_{n}$ to $\widetilde{M}$. These lifts are not necessarily closed but, for each $i=1, \ldots, n$, there is a covering transformation $g_{i} \in G$ relating the endpoints of $\gamma_{i}$ and, for $i, j=1, \ldots, n, g_{i}$ and $g_{j}$ are conjugate. Hence we may associate to $\gamma$ a well-defined conjugacy class $\langle\gamma\rangle \subset G$, called the Frobenius class of $\gamma$. These classes satisfy an analogue to Chebotarev's Theorem in number theory: for a conjugacy class $C \subset G$,

$$
\lim _{T \rightarrow+\infty} \frac{\#\{\gamma: l(\gamma) \leq T,\langle\gamma\rangle=C\}}{\#\{\gamma: l(\gamma) \leq T\}}=\frac{|C|}{|G|} .
$$

The identity (1.2) is proved by considering $L$-functions

$$
L\left(s, R_{\chi}\right)=\prod_{\gamma} \operatorname{det}\left(I-R_{\chi}(\langle\gamma\rangle) e^{-s l(\gamma)}\right)^{-1},
$$

where $R_{\chi}$ is an irreducible representation of $G$ with character $\chi$. Since $R_{\chi}$ lifts to a representation of $\Gamma, L\left(s, R_{\chi}\right)$ is a special case of the $L$-functions defined by (1.1). The geodesic flow on $S M$ is also covered by the geodesic flow on $S \widetilde{M}$, with covering group $G$, and hence the analytic properties of $L\left(s, R_{\chi}\right)$ may be deduced from the results in 8 .

Lemma 1.1. (i) Let $\mathbf{1}$ denote the trivial one-dimensional representation of $G$. Then $L(s, \mathbf{1})$ is analytic and nonzero on a neighbourhood of $\{s: \operatorname{Re}(s) \geq$ $h\}$, apart from a simple pole at $s=h$.

(ii) If $R_{\chi} \neq \mathbf{1}$ is an irreducible representation of $G$, then $L(s, \mathbf{1})$ is analytic and nonzero on a neighbourhood of $\{s: \operatorname{Re}(s) \geq h\}$.

In this paper, we shall refine (1.2) by requiring that $\gamma$ lies in a prescribed homology class in $H=H_{1}(M, \mathbb{Z})$. More precisely, for $\alpha \in H$, we shall write $\pi(T, \alpha)=$ $\#\{\gamma: l(\gamma) \leq T,[\gamma]=\alpha\}$ and $\pi(T, \alpha, C)=\#\{\gamma: l(\gamma) \leq T,[\gamma]=\alpha,\langle\gamma\rangle=C\}$ and study the ratio

$$
\frac{\pi(T, \alpha, C)}{\pi(T, \alpha)},
$$

where $[\gamma] \in H_{1}(M, \mathbb{Z})$ denotes the homology class of $\gamma$. Theorem 1 states that either $\pi(T, \alpha, C)$ is identically zero or (1.3) has a limit as $T \rightarrow+\infty$. We shall prove this in the next section; however, to do so, we need to first recall how $\pi(T, \alpha)$ behaves as $T \rightarrow+\infty$.

The asymptotics of $\pi(T, \alpha)$ are also obtained by considering a family of $L$ functions, in this case indexed by the characters of $H$. We suppose that $H$ is 
infinite and, for simplicity, we consider $H$ modulo torsion. Then these characters may be identified with the torus $\mathbb{T}^{d}=\mathbb{R}^{d} / \mathbb{Z}^{d}, d \geq 1$. For $\theta \in \mathbb{T}^{d}$, we write

$$
L(s, \theta)=\prod_{\gamma}\left(1-e^{-s l(\gamma)+2 \pi i \theta \cdot[\gamma]}\right)^{-1} .
$$

Since characters of $H$ lift to $\Gamma$, this is again an $L$-function of the form defined in (1.1).

We also write

$$
\eta_{\alpha}(s)=\int_{\mathbb{T}^{d}} e^{-2 \pi i \theta \cdot \alpha} \frac{d^{\nu+1}}{d s^{\nu+1}}(\log L(s, \theta)) d \theta,
$$

where $\nu=[d / 2]$. The following lemma is taken from [5] and [12].

Lemma 1.2. For each $\alpha \in H, \eta_{\alpha}(s)$ is analytic for $\operatorname{Re}(s)>h$.

(i) If $d$ is even, then, for some constant $c_{0}>0$,

$$
\lim _{\sigma \rightarrow h}\left(\eta_{\alpha}(\sigma+i \tau)-\frac{(-1)^{\nu+1} c_{0}}{\sigma+i \tau-h}\right)
$$

exists for almost every $\tau \in \mathbb{R}$ and is locally integrable. Moreover, there exists a locally integrable function $f(\tau)$ such that, for $\sigma>h$,

$$
\left|\eta_{\alpha}(\sigma+i \tau)-\frac{(-1)^{\nu+1} c_{0}}{\sigma+i \tau-h}\right| \leq f(\tau)
$$

(ii) If $d$ is odd, then, for some constant $c_{0}>0$,

$$
\lim _{\sigma \rightarrow h}\left(\eta_{\alpha}(\sigma+i \tau)-\frac{(-1)^{\nu+1} c_{0} \sqrt{\pi}}{\sqrt{\sigma+i \tau-h}}\right)
$$

exists for almost every $\tau \in \mathbb{R}$ and is locally integrable with locally integrable first derivative. Moreover, there exists a locally integrable function $f(\tau)$ such that, for $\sigma>h$,

$$
\left|\eta_{\alpha}(\sigma+i \tau)-\frac{(-1)^{\nu+1} c_{0} \sqrt{\pi}}{\sqrt{\sigma+i \tau-h}}\right| \leq f(\tau) .
$$

The constant $c_{0}$ in (i) and (ii) is independent of $\alpha$.

When combined with appropriate Tauberian theorems, this lemma is enough to ensure that, for some constant $c>0$, independent of $\alpha \in H$,

$$
\lim _{T \rightarrow+\infty} T^{1+d / 2} e^{-h T} \pi(T, \alpha)=c .
$$

(See [5] or [12] for more details.)

\section{Proof of Theorem 1}

It is clear that, in general, (1.3) will depend on the relationship between $G$ and $H$ and, particularly, $C$ and $\alpha$. Write $A=G /[G, G]$, the abelianization of $G$. Clearly, $A$ is a quotient of $\pi_{1}(M)$ and, since $H$ is the maximal abelian quotient of $\pi_{1}(M)$, $A$ is also a quotient of $H$. The extreme cases are:

(a) $G$ is abelian. Then $G=A$ and $G$ itself is a quotient of $H$.

(b) $G$ is perfect. Then $G=[G, G]$ and $A$ is trivial. 
We shall write $\pi_{G}: G \rightarrow A$ and $\pi_{H}: H \rightarrow A$ to denote the respective projections. In particular, it is clear that if $\pi_{G}(C) \neq \pi_{H}(\alpha)$, then $\pi(T, \alpha, C)=0$ for all $T>0$.

The proof of Theorem 1 depends on considering $L$-functions defined with respect to unitary representations of $\Gamma$ of the form $\theta \otimes R_{\chi}$, where $\theta \in \mathbb{T}^{d}$ and $R_{\chi}$ is an irreducible representation of $G$ (or, more precisely, the lifts of these quantities to $\Gamma)$. However, as we shall describe below, some of these $\theta \otimes R_{\chi}$ are trivial. The corresponding $L$-functions take the form

$$
\begin{aligned}
L\left(s, \theta \otimes R_{\chi}\right) & =\prod_{\gamma} \operatorname{det}\left(I-R_{\chi}(\langle\gamma\rangle) e^{-\operatorname{snl}(\gamma)+2 \pi i n \theta[\gamma]}\right) \\
& =\exp \sum_{\gamma} \sum_{n=1}^{\infty} \frac{1}{n} \chi\left(\langle\gamma\rangle^{n}\right) e^{-\operatorname{snl} l(\gamma)+2 \pi i n \theta[\gamma]},
\end{aligned}
$$

which converge to analytic functions for $\operatorname{Re}(s)>h$. Taking the logarithm and differentiating $\nu+1$ times gives

$$
\left(\frac{d}{d s}\right)^{\nu+1}(\log L)\left(s, \theta \otimes R_{\chi}\right)=\sum_{\gamma} \sum_{n=1}^{\infty} n^{\nu}(-l(\gamma))^{\nu+1} \chi\left(\langle\gamma\rangle^{n}\right) e^{-\operatorname{snl} l(\gamma)+2 \pi i n \theta \cdot[\gamma]} .
$$

Applying the standard orthogonality relations for both for irreducible representations of $G$ and for $\mathbb{T}^{d}$ term-by-term in the above formula we obtain the relation

$$
\begin{aligned}
& \sum_{R_{\chi} \text { irred }} \int_{\mathbb{T}^{d}} e^{-2 \pi i \theta \cdot \alpha} \bar{\chi}(C)\left(\frac{d}{d s}\right)^{\nu+1}(\log L)\left(s, \theta \otimes R_{\chi}\right) d \theta \\
& =-\frac{|G|}{|C|} \sum_{\substack{\gamma, n \\
n[\gamma]=\alpha,\left\langle\gamma^{n}\right\rangle=C}} n^{\nu} l(\gamma)^{\nu+1} e^{-s n l(\gamma),}
\end{aligned}
$$

where the right-hand side vanishes if $\pi_{G}(C) \neq \pi_{H}(\alpha)$.

The asymptotic behaviour of $\pi(T, \alpha, C)$ may be deduced from properties of the right-hand side of (2.1); so, to prove Theorem 1, it is enough to study

$$
\sum_{R_{\chi} \text { irred }} \int_{\mathbb{T}^{d}} e^{-2 \pi i \theta \cdot \alpha} \bar{\chi}(C)\left(\frac{d}{d s}\right)^{\nu+1}(\log L)\left(s, \theta \otimes R_{\chi}\right) d \theta
$$

and understand its meromorphic extension, via that of $L\left(s, \theta \otimes R_{\chi}\right)$, and, in particular, the nature of the singularites on $\operatorname{Re}(s)=h$.

First we determine which $\theta \otimes R_{\chi}$ are trivial. Let $m=|G /[G, G]|$ be the cardinality of $G /[G, G]$ and let $\mathbf{1}=\chi_{0}, \cdots, \chi_{m-1}$ be the characters of $G /[G, G]$ (i.e., the 1-dimensional representations of $G /[G, G]$ ). These lift to $G$ via $\pi_{G}$ but 1dimensional characters on $G$ also descend to $G /[G, G]$, since any such character annihilates commutators. Thus we may identify 1-dimensional representations of $G$ with characters of $G /[G, G]$. Each $\chi_{i}$ also lifts to a character of $H$, which we can denote by $\theta_{i}$.

Lemma 2.1. The representation $\theta \otimes R_{\chi}$ is trivial precisely when it is of the form $\theta_{i}^{-1} \otimes R_{\chi_{i}}, i=0, \ldots, m-1$.

Proof. It is clear from their construction that these representations are trivial. On the other hand, if $\theta \otimes R_{\chi}$ is trivial, then $R_{\chi}$ is one dimensional and hence it corresponds to one of the characters $\chi_{0}, \ldots, \chi_{m-1}$ of $G /[G, G]$. It is easy to see that, for $i=0, \ldots, m-1, \theta \otimes \chi_{i}$ is trivial only if $\theta=\theta_{i}^{-1}$. 
An immediate consequence is the following.

Lemma 2.2. For $i=0, \ldots, m-1$, the function $\zeta(s):=L\left(s, \theta_{i}^{-1} \otimes R_{\chi_{i}}\right)$ has a simple pole at $s=h$ and no other poles on $\operatorname{Re}(s)=h$.

Next we can consider $L\left(s, \theta \otimes R_{\chi}\right)$ for $\theta \otimes R_{\chi}$ nontrivial.

Lemma 2.3. If $L\left(s, \theta \otimes R_{\chi}\right)$ has a pole on $\operatorname{Re}(s)=h$, then $R_{\chi}$ is 1-dimensional and $\chi$ is a character of $G /[G, G]$, namely, one of the $\chi_{1}, \ldots, \chi_{m-1}$.

Proof. This follows from the discussion on page 146 of 8 .

If $R_{\chi}$ is 1-dimensional, then, as above, we can lift $\theta \otimes R_{\chi}$ to $\theta+\theta_{i} \in \mathbb{T}^{2 g}$ and rewrite the $L$-function as

$$
L\left(s, \theta \otimes R_{\chi}\right)=L\left(s, \theta+\theta_{i}\right)=\prod_{\gamma}\left(1-e^{-s l(\gamma)+2 \pi i\left(\theta+\theta_{i}\right) \cdot[\gamma]}\right)^{-1} .
$$

However, this is again an $L$-function for homology.

Lemma 2.4. If $\theta \otimes R_{\chi}$ is nontrivial, then $L\left(s, \theta \otimes R_{\chi}\right)$ is analytic on $\operatorname{Re}(s)=h$.

Proof. By the above, we only need to consider the case when $L\left(s, \theta \otimes R_{\chi}\right)=L(s, \theta+$ $\left.\theta_{i}\right)$. However, if $\theta \otimes R_{\chi}$ is nontrivial, then $\theta \neq-\theta_{i}$, so the lemma follows from standard results in [3], [5], 10], 12].

To proceed, we return to the expression (2.1). We can rewrite this as

$$
\frac{|C|}{|G|} \sum_{i=0}^{m-1} \overline{\chi_{i}}(C) \int_{\mathbb{T}^{2 g}} e^{-2 \pi i \theta \cdot \alpha}\left(\frac{d}{d s}\right)^{g+1}(\log L)\left(s, \theta \otimes R_{\chi_{i}}\right) d \theta+\phi(s),
$$

where $\phi(s)$ is a function analytic in a neighbourhood of $\operatorname{Re}(s)=h$ and, from (2.2), $L\left(s, \theta \otimes R_{\chi_{i}}\right)=L\left(s, \theta+\theta_{i}\right)$. We also note that, since we are assuming that $\pi_{G}(C)=\pi_{H}(\alpha)$, we have that

$$
\bar{\chi}_{i}(C) e^{-2 \pi i\left(-\theta_{i}\right) \cdot \alpha}=1, \text { for } i=0, \ldots, m-1 .
$$

Hence one sees that the function in (2.1) satisfies an analogue of Lemma 1.2 in which that $c_{0}$ is replaced by $c_{0} m|G| /|C|$.

From this one deduces, as in [5] or [12], that

$$
\lim _{T \rightarrow+\infty} T^{1+d / 2} e^{-h T} \pi(T, \alpha, C)=c m \frac{|C|}{|G|},
$$

with $c$ as in (1.4).

Finally, recalling that $m=|G /[G, G]|$, this is enough to prove Theorem 1 .

Remarks. (i) If $M$ is either a surface or has curvature that is 1/4-pinched, then, working along the lines of [1], 6], 11], one can get an $O\left(T^{-1}\right)$ error term, as Zelditch obtained for a hyperbolic surface. It is also possible to prove analogous results where a fixed homology class is replaced by one which changes linearly in $T$ (cf. [2, 7]).

(ii) There is a natural extension of Theorem 1 to Anosov flows which are homologically full in the sense of [12, i.e., ones for which every homology class is represented by a periodic orbit. 


\section{Proof of Theorem 2}

We can easily adapt the proof of Theorem 1 to prove Theorem 2 . Since we are replacing a finite group $G$ by a compact group $S O(n-1)$ we need to consider a countable family of representations $R_{\chi}$, rather than a finite family. However, by approximation it suffices to consider each representation separately. As in the proof in the last section, one can consider representations $\theta \otimes R_{\chi}$. However, a significant advantage here is that the groups $H$ and $S O(n-1)$ can be treated independently.

In the case of the trivial representation, we have that $F=\chi=\mathbf{1}$ and we see that

$$
\left(\frac{d}{d s}\right)^{\nu+1}(\log L)(s, \theta \otimes \mathbf{1})
$$

has a singularity of the form

$$
\text { Const. } \times \frac{1}{(s-s(\theta))} .
$$

The analysis reduces to that in [5], [12, from which we get an asymptotic formula

$$
\lim _{T \rightarrow+\infty} \frac{1}{\pi(T, \alpha)} \sum_{\substack{l(\gamma) \leq T \\ \gamma=\alpha}} F(\Theta(\gamma))=1 .
$$

However, in the case of nontrivial representations we have that the $L$-function $L(s, \theta \otimes \mathbf{1})$ is always analytic on $\operatorname{Re}(s)=h$, from which one sees that

$$
\sum_{\substack{l(\gamma) \leq T \\ \gamma=\alpha}} F(\Theta(\gamma))=o(\pi(T, \alpha)) .
$$

\section{REFERENCES}

1. N. Anantharaman, Precise counting results for closed orbits of Anosov flows, Ann. Sci. École Norm. Sup. 33 (2000), 33-56. MR.1743718 (2002c:37048)

2. M. Babillot and F. Ledrappier, Lalley's theorem on periodic orbits of hyperbolic flows, Ergodic Theory Dynam. Systems 18 (1998), 17-39. MR.1609507 (99a:58128)

3. A. Katsuda, Density theorems for closed orbits, Geometry and analysis on manifolds (KatataKyoto, 1987), Lecture Notes in Math., 1339, Springer, Berlin, 1988, pp. 182-202. MR.961481 (89j:58066)

4. A. Katsuda and T. Sunada, Homology and closed geodesics in a compact Riemann surface, Amer. J. Math. 110 (1988), 145-155. MR.926741(89e:58093)

5. A. Katsuda and T. Sunada, Closed orbits in homology classes, Inst. Hautes Études Sci. Publ. Math. 71 (1990), 5-32. MR.1079641 (92m:58102)

6. M. Kotani, A note on asymptotic expansions for closed geodesics in homology classes, Math. Ann. 320 (2001), 507-529. MR1846775 (2002h:58044)

7. S. Lalley, Closed geodesics in homology classes on surfaces of variable negative curvature, Duke Math. J. 58 (1989), 795-821. MR1016446 (91a:58143)

8. W. Parry and M. Pollicott, The Chebotarev theorem for Galois coverings of Axiom A flows, Ergodic Theory Dynam. Systems 6 (1986), 133-148. MR837980 (88k:58124)

9. R. Phillips and P. Sarnak, Geodesics in homology classes, Duke Math. J. 55 (1987), 287-297. MR $894581(88 \mathrm{~g}: 58151)$

10. M. Pollicott, Homology and closed geodesics in a compact negatively curved surface, Amer. J. Math 113 (1991), 379-385. MR1109342 (92e:58158)

11. M. Pollicott and R. Sharp, Asymptotic expansions for closed orbits in homology classes, Geom. Ded. 87 (2001), 123-160. MR1866845 (2003b:37051)

12. R. Sharp, Closed orbits in homology classes for Anosov flows, Ergodic Theory Dynam. Systems 13 (1993), 387-408. MR.1235480 (94g:58169) 
13. T. Sunada, Geodesic flows and geodesic random walks, Geometry of geodesics and related topics (Tokyo, 1982), Adv. Stud. Pure Math., 3, North-Holland, Amsterdam, 1984, pp. 47-85. MR 758647 (86i:58104)

14. S. Zelditch, Splitting of geodesics in homology classes, Proc. Amer. Math. Soc. 105 (1989), 1015-1019. MR946640 (89k:58236)

Department of Mathematics, University of Warwick, Coventry, CV4 7AL, United KINGDOM

E-mail address: mpollic@maths.warwick.ac.uk

School of Mathematics, University of Manchester, Oxford Road, Manchester M13 9PL, UNITED KINGDOM

E-mail address: sharp@maths.man.ac.uk 